\title{
Analytical prediction of void distribution and a minimum- void angle in anisotropic fabrics for radial injection resin transfer molding
}

\author{
R. Matsuzaki ${ }^{*}$, M. Naito ${ }^{1}$, D. Seto ${ }^{2}$, A. Todoroki $^{2}$, Y. Mizutani $^{2}$ \\ ${ }^{1}$ Department of Mechanical Engineering, Tokyo University of Science, 2641 Yamazaki, Noda, 278-8510 Chiba, Japan \\ ${ }^{2}$ Department of Mechanical Sciences and Engineering, Tokyo Institute of Technology, 2-12-1 O-okayama, Meguro, \\ 152-8552 Tokyo, Japan
}

Received 20 March 2016; accepted in revised form 5 June 2016

\begin{abstract}
D radial injection vacuum-assisted resin transfer molding experiments were performed using anisotropic plainwoven fabrics to determine the void distribution and the relationship between the void fraction and the resin flow velocity at arbitrary resin impregnation angles. The obtained void fraction values vary with the impregnation angle and velocity, while void formation is very difficult at the minimum-void angle oriented in neither the warp nor the weft direction. Moreover, the impregnation in the fabric microscopic structure is characterized by two patterns separated at the minimum-void angle. Based on the experimental results, a mathematical model for predicting the void fraction value at arbitrary impregnation angles and velocities and for calculating the minimum-void angle was developed. A comparison of the model predictions with the experimental results revealed a good agreement between them.
\end{abstract}

Keywords: polymer composites, processing technologies, modeling and simulation, resin transfer molding (RTM), defects

\section{Introduction}

Vacuum-assisted resin transfer molding (VaRTM) has attracted attention because of its capability to replace the autoclave molding method with a low-cost composite material molding method [1-3]. However, resin impregnation of the VaRTM may be insufficient due to the inhomogeneity of the fabrics used for reinforcement $[4,5]$, resulting in the formation of cavities or voids, which decrease the strength of the final product [6-8]. The voids formed during the impregnation may be classified as macro-scale voids, mesoscale voids or micro-scale voids depending on their size. A macro-scale void is also called a dry spot and is formed in the domain where the fabric is not impregnated by the resin because of inappropriate molding conditions [9-11]. A meso-scale void is caused by inter-bundle air trapping resulting from the differ- ences between the high value of the intra-bundle resin velocity and the low value of the inter-bundle resin velocity [12-14]. A micro-scale void is formed by the air trapped in the bundles due to the differences between the low value of the intra-bundle resin flow velocity and the high value of the inter-bundle resin flow velocity $[13,15,16]$.

Various studies have been conducted on the prevention of void formation. To prevent the appearance of macro-scale voids, various simulations were performed to determine the optimal gate location [17-20]. Unlike the macro-scale voids, meso-scale and microscale voids are caused by the unevenness of the fabric microstructure, which depends on the capillary number, and numerical models for predicting the void fraction values have been developed in previous studies [13, 21, 22]. Park et al. [21] proposed a method

${ }^{*}$ Corresponding author, e-mail: rmatsuza@rs.tus.ac.jp

(C) BME-PT 
for predicting the meso-scale and micro-scale void fractions by calculating the intra-bundle and interbundle flow velocities. Matsuzaki et al. [23] presented a model for predicting the meso-scale void-fraction in the warp and weft directions of anisotropically woven fabrics. They constructed a model for predicting the amount of void formation based on the voidformation process observed in the experiment and compared the void-formation estimation and measurement results. However, these previous studies neither developed nor validated a prediction model that takes into account the effects produced by the angle and velocity of the resin impregnation on void formation in an anisotropic or any other type of fabric; only the relationship between the resin flow velocity and void formation during $1 \mathrm{D}$ resin impregnation was investigated. During resin impregnation in a real structure, the resin flows through the fabric at an arbitrary angle and velocity owing to the complexity of the fabric structure and the effects of the resin infusion point. Therefore, it is necessary to evaluate the amount of void formation in relation to the impregnation angle and resin velocity because void formation is thought to be predicted by estimating the amount of void formation in relation to the resin velocity for impregnation in an arbitrary direction, with the actual impregnation being not just in the warp and weft directions. In addition, the resin impregnation path through the microscopic structure of the fabric may change with the resin impregnation angle, and there may possibly be an optimal value of the angle that reduces the void fraction compared to when the resin is injected in the warp or the weft direction.

The purpose of this study is to evaluate the effect of the resin impregnation angle in an anisotropic fabric on the relationship between the amount of voids formed and the resin velocity. We performed exper-

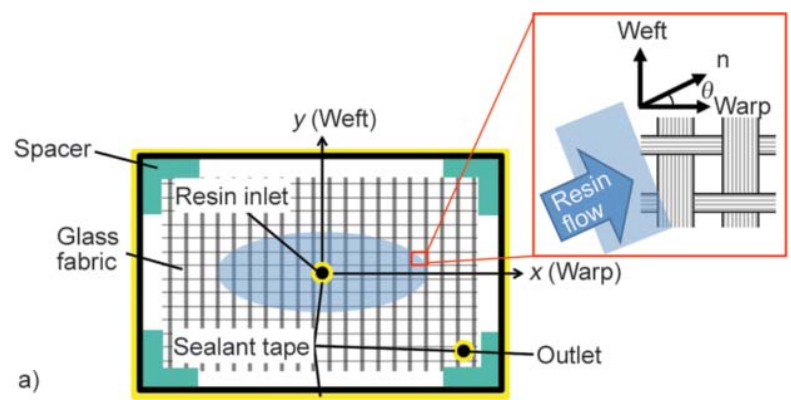

imental 2D radial-injection VaRTM and investigated the meso-scale void fraction distribution and the void formation mechanism for one-layered anisotropically woven fabrics. Henceforth in this paper, the word 'void' refers to a meso-scale void. Based on the experimental results, we also developed an analytical model for predicting the void fraction for an arbitrary resin impregnation angle and velocity, and for determining the optimal minimum-void angle. The developed model was validated by comparing its predictions with the experimental results. It should be noted that because the subject of the present study is plainwoven fabric, the model presented is for fabric that has sufficiently large inter-bundle cavities for which the void fraction can be estimated if the size of the voids formed in inter-bundle cavities exceeds the thickness of the fabric.

\section{Void observations in the $2 D$ radial-injection VaRTM}

\subsection{D radial-injection VaRTM}

To study the relationship between the resin flow velocity and the void fraction during resin impregnation in a fabric at an arbitrary angle, an experimental 2D radial-injection VaRTM was performed from the fabric center. Figure 1 shows a schematic of the experimental device used in this work. A one-layer glass fabric was inserted between the upper and the bottom glass molds. The circumference of the mold was sealed with a sealant tape, while the glass mold was fixed with a vice. Spacers made from a $0.3 \mathrm{~mm}$ thick glass-fiber reinforced plastic were used to adjust the gap between the glass molds, while the thickness of the fabric was maintained constant. The pressure in the vacuum pump was controlled by a pilot-type vacuum regulator (Koganei, NVR200-01). Table 1 lists the material properties of the two fabrics used in the

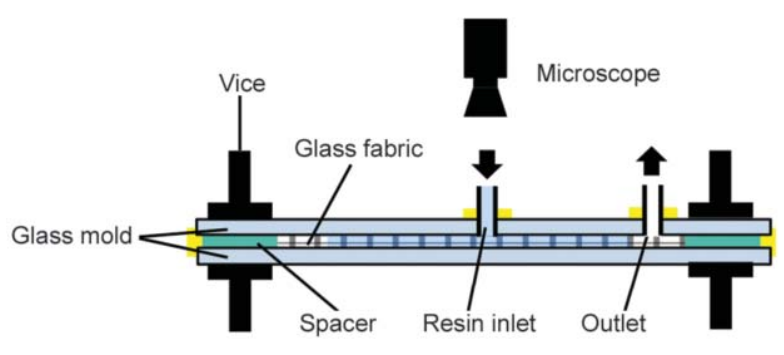

b)

Figure 1. A schematic of the 2D VaRTM experiment. (a) Top view: the resin is impregnated radially from the center. (b) Side view: the fabric is laid between the transparent glass boards. The void fraction and void distribution are observed by a microscope and a camera. 
Table 1. Material properties of the fabrics used in this work

\begin{tabular}{|l|c|c|}
\hline \multirow{2}{*}{\multicolumn{1}{|c|}{ Property }} & \multicolumn{2}{c|}{ Value (SD) } \\
\cline { 2 - 3 } & \multicolumn{2}{c|}{ M155 } \\
\hline Macroscopic porosity, $\Phi[\%]$ & $19.6( \pm 0.68)$ & $15.2( \pm 0.62)$ \\
\hline Height of a fiber bundle in the warp direction, $h_{\text {warp }}[\mathrm{m}]$ & $1.50 \cdot 10^{-4}\left( \pm 0.04 \cdot 10^{-4}\right)$ & $1.8 \cdot 10^{-4}\left( \pm 0.04 \cdot 10^{-4}\right)$ \\
\hline Height of a fiber bundle in the weft direction, $h_{\text {weft }}[\mathrm{m}]$ & $1.10 \cdot 10^{-4}\left( \pm 0.04 \cdot 10^{-4}\right)$ & $1.2 \cdot 10^{-4}\left( \pm 0.13 \cdot 10^{-4}\right)$ \\
\hline Distance between the bundles in the warp direction, $l_{\text {warp }}[\mathrm{m}]$ & $4.80 \cdot 10^{-4}\left( \pm 0.27 \cdot 10^{-4}\right)$ & $4.2 \cdot 10^{-4}\left( \pm 0.26 \cdot 10^{-4}\right)$ \\
\hline Distance between the bundles in the weft direction, $l_{\text {weft }}[\mathrm{m}]$ & $6.50 \cdot 10^{-4}\left( \pm 0.41 \cdot 10^{-4}\right)$ & $6.8 \cdot 10^{-4}\left( \pm 0.38 \cdot 10^{-4}\right)$ \\
\hline Macroscopic permeability in the warp direction, $K_{\mathrm{x}}\left[\mathrm{m}^{2}\right]$ & $4.01 \cdot 10^{-10}\left( \pm 3.94 \cdot 10^{-11}\right)$ & $4.49 \cdot 10^{-10}\left( \pm 1.62 \cdot 10^{-10}\right)$ \\
\hline Macroscopic permeability in the weft direction, $K_{\mathrm{y}}\left[\mathrm{m}^{2}\right]$ & $5.39 \cdot 10^{-11}\left( \pm 5.73 \cdot 10^{-12}\right)$ & $5.61 \cdot 10^{-11}\left( \pm 2.72 \cdot 10^{-11}\right)$ \\
\hline Width of a fiber bundle in the warp direction, $w_{\text {warp }}[\mathrm{m}]$ & $5.70 \cdot 10^{-4}\left( \pm 0.16 \cdot 10^{-4}\right)$ & $6.9 \cdot 10^{-4}\left( \pm 0.32 \cdot 10^{-4}\right)$ \\
\hline Width of a fiber bundle in the weft direction, $w_{\text {weft }}[\mathrm{m}]$ & $1.00 \cdot 10^{-3}\left( \pm 0.53 \cdot 10^{-4}\right)$ & $1.1 \cdot 10^{-3}\left( \pm 0.49 \cdot 10^{-4}\right)$ \\
\hline Porosity inside a bundle, $\varphi_{\mathrm{f}}[\%]$ & $48( \pm 8.1)$ & $48( \pm 8.1)$ \\
\hline Fiber radius $r[\mu \mathrm{m}]$ & 4.5 & 4.5 \\
\hline
\end{tabular}

experiments: M155K104 and M200K104 (Unitika Glass Fiber; henceforth referred to as M155 and M200, respectively; see also Figure 2). The fiber bundle of M200 was bigger than that of M155, while the macroscopic cavity ratio of the former material was smaller. The material properties of the unsaturated polyester resin (DH material, Sundhoma PC184-C) are listed in Table 2. In addition, the impregnation angle of the resin was set to $0^{\circ}$ for the warp direction of the fabric and to $90^{\circ}$ for the weft direction. In Figure 1a, the resin impregnation direction is denoted by $\mathbf{n}$. The resin impregnation angle $\theta$ was defined as the angle between the warp direction and the resin impregnation direction $\mathrm{n}$. The vacuum pressure $P_{\text {vac }}$ was set to $50 \mathrm{kPa}$ for M155 and $30 \mathrm{kPa}$ for M200 during the experiments. In a preliminary experiment, micro-scale void formation was hardly observed. Vacuum pressures under which meso-scale void formation was likely to be observed were sought out in the

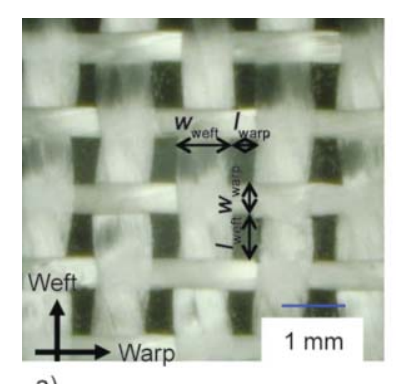

a)

Figure 2. Fabrics used for the experiments in this work: (a) M155, (b) M200

Table 2. Material properties of the resin at $25^{\circ} \mathrm{C}$

\begin{tabular}{|l|c|}
\hline \multicolumn{1}{|c|}{ Property } & Value \\
\hline Viscosity, $\mu[\mathrm{Pa} \cdot \mathrm{s}]$ & 0.169 \\
\hline $\begin{array}{l}\text { Product of the surface energy } \gamma \text { and the cosine of the con- } \\
\text { tact angle } \theta_{\mathrm{c}},\left(\cos \theta_{\mathrm{c}}\right)[\mathrm{Nm}]\end{array}$ & 0.025 \\
\hline
\end{tabular}

respective fabrics, and the experiments were performed at the appropriate pressure.

To determine the void distribution for an arbitrarily set resin impregnation angle in the fabric, we used a camera to photograph the entire material after the completion of the resin impregnation process. The $x$ axis and the $y$ axis were defined as the warp and weft directions, respectively, with the origin at the resin inlet point. Because the resin impregnation was performed radially it was assumed to be symmetrical, and the measurements were only conducted in the domain of $x>0$ and $y>0$. To evaluate the effect of the impregnation direction on the relationship between the resin flow velocity and the void fraction, the void fraction value was determined at different impregnation angles. A microscope (Keyence VHX900) was used to photograph the fabrics after the flow front had passed at an arbitrary angle. As shown in Figures 3, the photographic images were digitally processed to extract the outline of the voids and measure the void fraction. The voids are columnar in shape, thus it was assumed that the $2 \mathrm{D}$ void fraction value

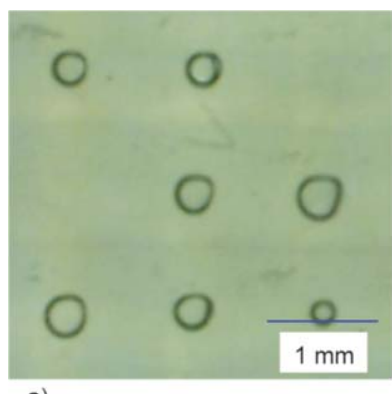

a)

Figure 3. A method of measuring the void fraction: (a) voids observed with a microscope, (b) an image analysis of the obtained voids to determine the void fraction value 
measured by means of the image analysis was equal to the volumetric void fraction [24].

\subsection{Experimental results and discussion}

Figures 4 show the resin impregnation and void formation in the microscopic structures of the M200 fabric at impregnation angles of 30 and $75^{\circ}$, respectively. The domains surrounded by the two warp bundles and two weft bundles are indicated by the red dotted lines. For both impregnation angles, the voids formed by the air trapping between the bundles were due to the fingering caused by the difference between the interbundle and intra-bundle resin flow velocities. In addition, the air trapping at the $30^{\circ}$ impregnation angle was due to the completion of the resin impregnation in the weft bundle after that in the warp bundle. In contrast, the air trapping at the $75^{\circ}$ impregnation angle was due to the completion of the resin impregnation in the warp bundle after that in the weft bundle. These observations confirm that the pattern of the intra-bundle resin impregnation vary with the impregnation angle. It should be noted that the formation of an undulating flow front is not considered problematic, because when observed microscopically, it can be regarded as impregnation at an oblique angle in the normal direction of the flow front. Moreover, the resin impregnation of crimps is extremely fast owing to the high fiber content and high capillary pressure. Furthermore, because crimps are present in both warp and weft fiber bundles, there is little impact on the resin impregnation when voids are shaped.
Figures $5 \mathrm{a}$ and $5 \mathrm{~b}$ show the void distributions in the M155 and M200 fabrics, respectively. The black points represent the voids, and the white part corresponds to the domain of the impregnated resin. The figures show that it is very difficult for voids to appear at the minimum-void angle value $\theta_{\min }$. The 'minimum-void angle' was obtained by measuring, in the void distribution images, the direction where the void distribution was the smallest. Moreover, the change in the void formation pattern occurs at the minimum-void angle (as observed in Figure 4). From the void distributions, the minimum-void angle $\theta_{\min }$ was determined to be approximately $49^{\circ}$ for $\mathrm{M} 155$ and $53^{\circ}$ for M200. Figures $5 \mathrm{c}$ and $5 \mathrm{~d}$ show the relationships between the void fractions and the capillary number $\mathrm{Ca}^{*}$ for M155 and M200, respectively. The void fraction was measured at resin impregnation angles of $0,30,75$, and $90^{\circ}$, and $\theta_{\min }$ values of $49^{\circ}$ for M155 and $53^{\circ}$ for M200. The results shown in the figure indicate that the void fraction increases with decreasing the macroscopic resin flow velocity regardless of the impregnation angle; this happens because the difference between the intra-bundle and inter-bundle flow velocities increases with decreasing the macroscopic flow velocity resulting in an increase in the trapped air volume [24-26]. In addition, the resin flow velocity at the beginning of void formation (i.e. the flow velocity when air gets trapped between bundles and voids start to form) was highest at an impregnation angle of $0^{\circ}$ and lowest at the minimum-void angle $\theta \mathrm{min}$ for both fabrics. The reason for the difference in the velocity of

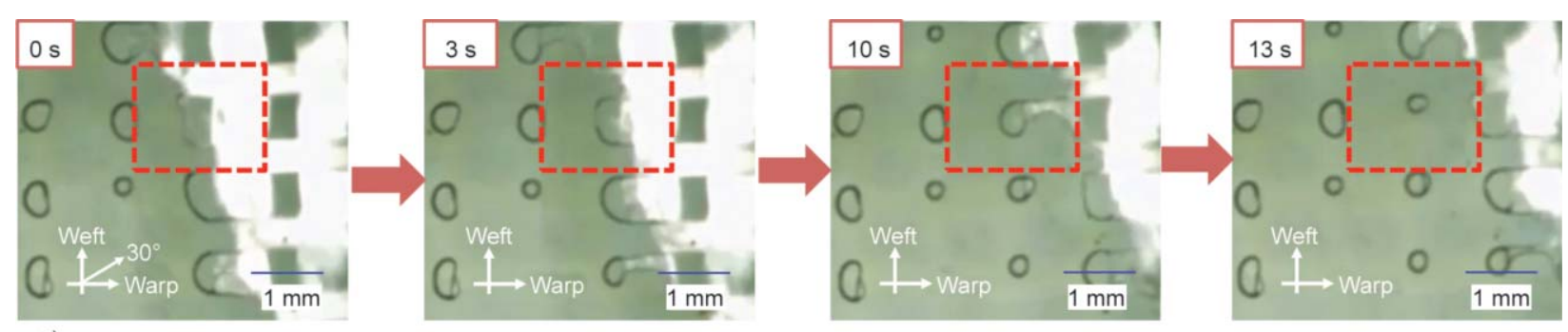

a)

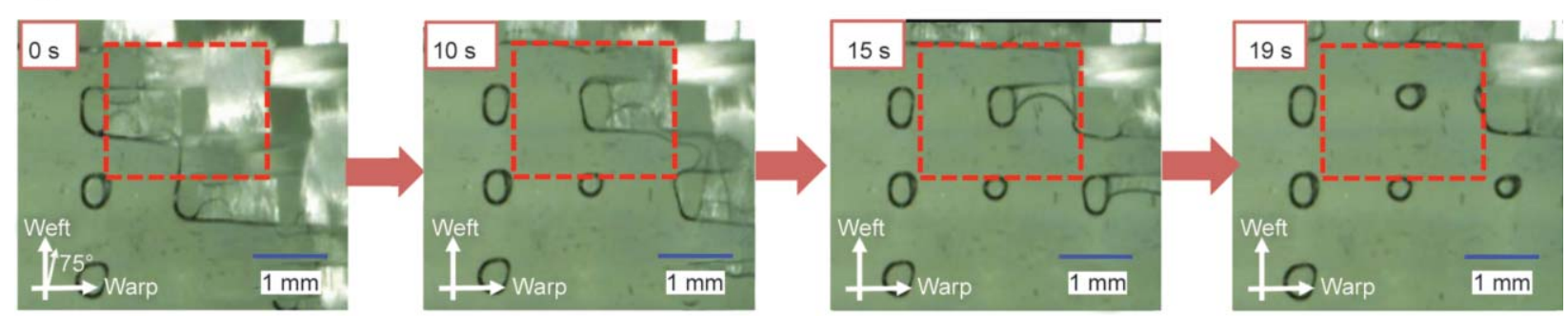

b)

Figure 4. Resin impregnation and void formation in the microscopic structure of M200 as observed with a microscope at impregnation angles of (a) $30^{\circ}$ and (b) $75^{\circ}$ 

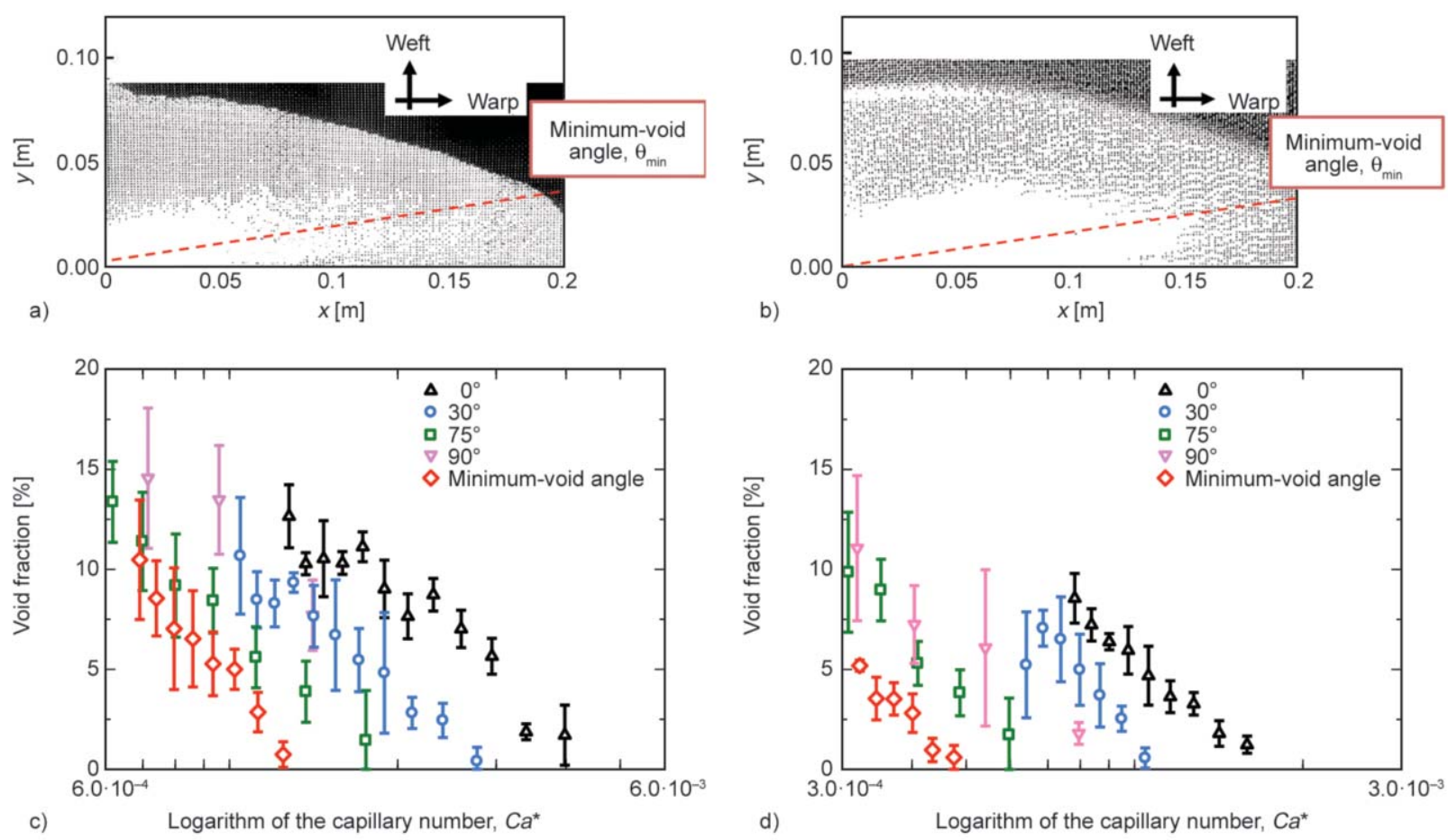

Figure 5. An observed void fraction distribution in the 2D radial injection VaRTM. The black points indicate voids, and the red line represents the impregnation angle corresponding to the minimum void fraction: (a) M155, (b) M200. An experimentally determined relationship between the capillary number and the void fraction at different flow directions: (c) M155, (d) M200.

void formation depending on the impregnation angle is attributed to the fact that the time required for intra-bundle and inter-bundle resin impregnation varies with the changes in the impregnation path length. The variation in the length of the intra-bundle impregnation path is larger than that of the length of the inter-bundle impregnation distance, as the impregnation angle inclines at an angle ranging from 0 to $90^{\circ}$. Moreover, because the physical properties and the macroscopic infiltration coefficient of the fiber bundles differ in the warp and weft directions, the timing of the void formation varies.

\section{Analytical model for the void fraction prediction}

\subsection{Void formation}

Figure 6 depicts a unit cell of the fabric used for the development of the void fraction prediction model. The unit cell is representative of the $2 \mathrm{D}$ composition of the fabric and contains two weft bundles and two warp bundles. In general, the unit cell was defined to consist of bundles, crimps, and an inter-bundle cavity.

The resin impregnation process of the unit cell used for developing the void fraction prediction model is

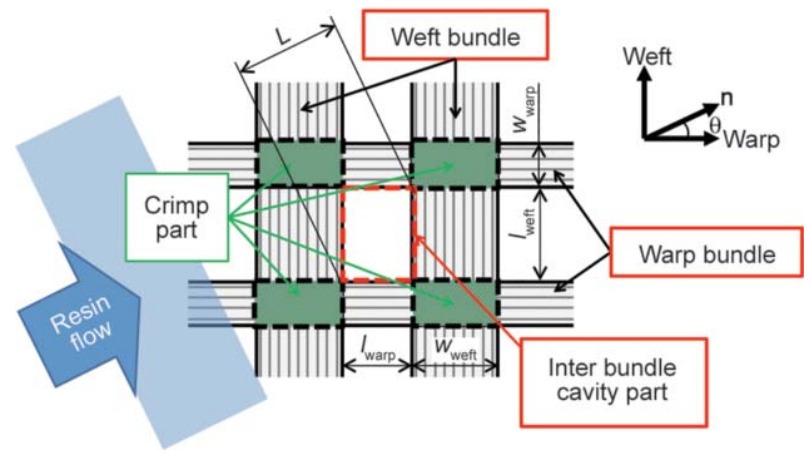

Figure 6. A unit cell of the woven fabric used for the void fraction prediction model

shown in Figure 7. The first stage shown in Figure 7a corresponds to the start of the impregnation process $(0 \mathrm{~s}$, Figure $4 \mathrm{a})$, and the macroscopic flow front reaches one of the crimps in the unit cell. At this time, the macroscopic flow front makes contact with the lower fiber bundle at the red dotted line shown in Figure 7a. The resin impregnation thereafter progresses to the second stage shown in Figure $7 \mathrm{~b}$, which corresponds to the image at $10 \mathrm{~s}$ in Figure 4a. At this stage, resin impregnation in the transverse fiber bundle on the upstream side of the unit cell is completed, and the impregnation in the inter-bundle cavity begins as indicated by the red dotted line in Figure $7 \mathrm{~b}$. The resin im- 

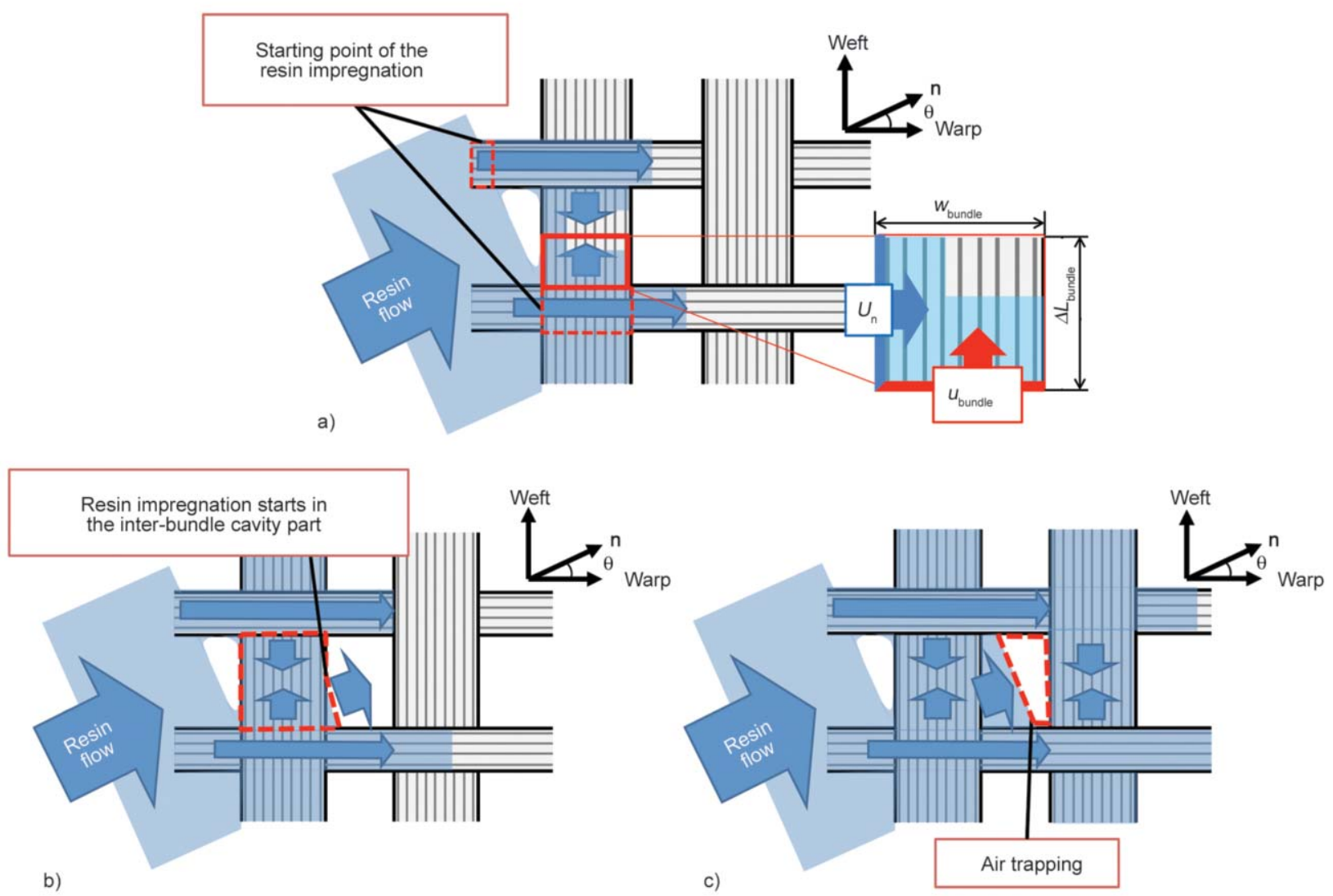

Figure 7. Stages of the resin impregnation in the unit cell: (a) Phase 1. Impregnation begins in the unit cell; the mechanism of the transverse bundle impregnation is shown. (b) Phase 2. After the completion of the transverse bundle impregnation, the resin impregnation of the inter-bundle cavity begins. (c) Phase 3. The intra-bundle resin impregnation is complete, and the air trapping process begins.

pregnation then progresses to the third stage shown in Figure 7c, which corresponds to the image at $19 \mathrm{~s}$ in Figure 4a. The intra-bundle resin impregnation in the unit cell is completed earlier than the inter-bundle impregnation due to the difference between the

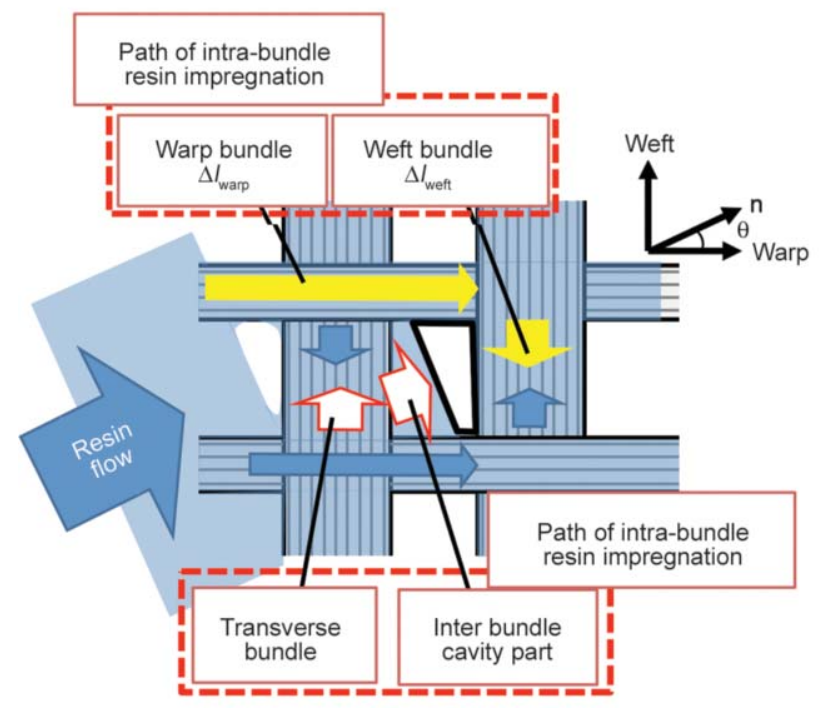

a) respective resin flow velocities. After the completion of the intra-bundle resin impregnation, the progress of the inter-bundle impregnation ceases, and the air trapped in the inter-bundle cavity forms a void. It should be noted that the inter-bundle impregnation

Figure 8. Descriptions of the impregnation paths in the unit cell: (a) Pattern 1, (b) Pattern 2 
path was modeled as the impregnation of the upstream transverse bundle and the impregnation of the inter-bundle cavity surrounded by fiber bundles. Based on the void formation pattern for each impregnation angle in Figure 4, the impregnation paths were determined for Patterns 1 and 2 corresponding to the small and large impregnation angles, respectively, as shown in Figure 8. Detailed impregnation path modeling for each pattern will be performed in Sections 3.2 and 3.3 .

\subsection{Intra-bundle impregnation path}

An intra-bundle resin flow velocity for each bundle (warp or weft) is defined by Equation (1), which is based on the Darcy's law with an additional term for the capillary pressure gradient $[21,25]$ :

$u=-\frac{k}{\mu \varphi_{\mathrm{cr}}}\left(\frac{\partial P}{\partial n_{\mathrm{axis}}}-\frac{P_{\mathrm{c}}}{\Delta l}\right)$

where $\mu$ is the viscosity of the resin, $P_{\mathrm{c}}$ is the capillary pressure in the intra-fiber bundles, $\varphi_{\mathrm{cr}}$ is the crimp porosity, $\Delta l$ is the intra-bundle impregnation distance. $\partial P / \partial n_{\text {axis }}$ denotes the macroscopic pressure gradients in the warp and weft bundle directions defined by Equations (2) and (3), respectively:

$\frac{\partial P}{\partial n_{\text {axis, warp }}}=\frac{\partial P}{\partial n} \cos \theta$

$\frac{\partial P}{\partial n_{\text {axis, weft }}}=\frac{\partial P}{\partial n} \sin \theta$

where $\partial P / \partial n$ is the macroscopic pressure gradient in the resin impregnation direction. $k$ is the permeability of each warp and weft bundle depending on the crimp permeability [27]. The crimp permeability is determined by the ratio of the longitudinal fiber bundle thickness to the transverse bundle thickness as shown in Figure 9a as well as the by the permeabilities of the

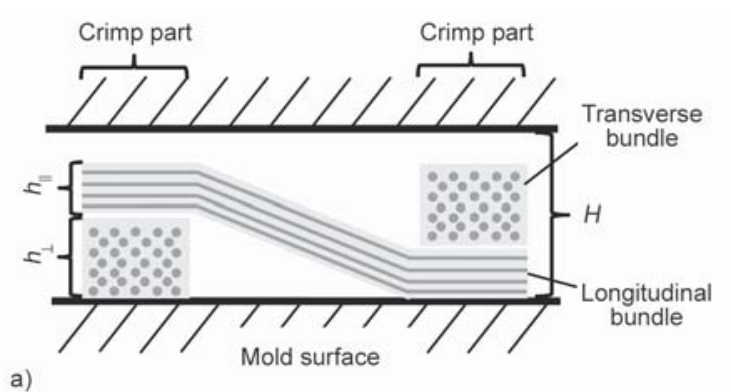

warp and weft bundles in both directions (Equation (4)):

$k=\frac{h_{\|} k_{\|}+h_{\perp} k_{\perp}}{h_{\|}+h_{\perp}}$

where $h_{\|}$and $h_{\perp}$ are the heights of each bundle in the bundling and transverse directions, respectively, and $k_{\|}$and $k_{\perp}$ are the permeabilities of the fiber bundles in the axial and transverse directions determined by the Gebart model as expressed by Equations (5) and (6) [28]:

$k_{\|}=\frac{8 r_{\mathrm{f}}^{2}}{C_{\|}} \cdot \frac{\varphi_{\mathrm{cr}}^{3}}{\left(1-\varphi_{\mathrm{cr}}\right)^{2}}$

$k_{\perp}=C_{\perp} r_{\mathrm{f}}^{2}\left(\sqrt{\frac{V_{\mathrm{f} \max }}{1-\varphi_{\mathrm{cr}}}}-1\right)^{\frac{5}{2}}$

where $r_{\mathrm{f}}$ is the radius of the fiber filament, and $C_{\|}$, $C_{\perp}$, and $V_{\mathrm{f} \text { max }}$ are the shape functions. The fibers are assumed to be hexagonally arranged; hence, $C_{\|}=$ 53, $C_{\perp}=16 /(9 \pi \sqrt{6})$, and $V_{\mathrm{f} \max }=\pi / \sqrt{6}$. In addition, the fabric section corresponding to the gap between the molds is homogenized to form the 2D model shown in Figure 9b. $\varphi_{\mathrm{cr}}$ is the average porosity of the gaps between the molds in the crimps defined by Equation (7):

$\varphi_{\mathrm{cr}}=1-\frac{h_{\|}+h_{\perp}}{H} \cdot\left(1-\varphi_{\mathrm{f}}\right)$

where $H$ is the height of the gap between the molds, and $\varphi_{\mathrm{f}}$ is the porosity of the fiber bundles. It should be noted that the porosity of the fiber bundles is assumed to be the same in both directions [23, 24].

In addition, the capillary pressure in Equation (1) is determined using the Young-Laplace equation represented by Equation (8) [29]:

$P_{c}=\frac{2 \varphi C \cos \theta_{\mathrm{c}}}{r_{\mathrm{c}}}$

b)

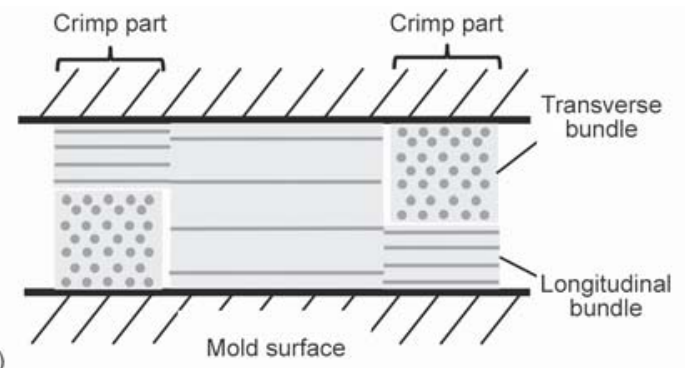

Figure 9. An effect of the thickness in the bundling direction on the superficial intra-bundle zone: (a) a realistic model, (b) an ideal model 
where $\gamma$ is the surface tension of the resin, $C$ is the geometric correction factor, $\theta_{\mathrm{c}}$ is the contact angle between the resin and the fibers, and $r_{\mathrm{c}}$ is the capillary radius of the fiber bundles specified by Equation (9). The geometric correction factor $C$ is obtained by fitting the data from the measurement results in the voidfraction experiments with the results acquired using the estimated model. The average porosity in the gaps between the molds $\varphi$ is defined by Equation (10):

$$
\begin{aligned}
& r_{\mathrm{c}}=\frac{\varphi}{1-\varphi} r_{\mathrm{f}} \\
& \varphi=1-\frac{h}{H}\left(1-\varphi_{\mathrm{f}}\right)
\end{aligned}
$$

where $h$ is the height of the bundles in the warp or weft direction.

The intra-bundle impregnation distance $\Delta l$ is different for Patterns 1 and 2 as indicated by the yellow arrows in Figure 8. Although both longer and shorter intra-bundle impregnation paths exist, the longer impregnation path was used to calculate the intra-bundle impregnation distance because it determines the intra-bundle impregnation time. Moreover, it was assumed for simplicity that the two intra-bundle impregnation fronts contact each other at the middle point of the downstream transverse bundle. The intra-bundle impregnation distances for the two patterns are determined by Equatios (11) and (12):

Pattern 1: $\theta<\theta_{\min }$

$\Delta l_{\text {warp }}=l_{\text {warp }}+l_{\text {weft }} \cdot \tan \theta, \Delta l_{\text {weft }}=\frac{l_{\text {weft }}}{2}$

Pattern 2: $\theta>\theta_{\min }$

$$
\Delta l_{\text {warp }}=\frac{l_{\text {warp }}}{2}, \Delta l_{\text {weft }}=l_{\text {weft }}+l_{\text {warp }} \cdot \tan \left(\frac{\pi}{2}-\theta\right)
$$

By integrating Equation (1), the intra-bundle impregnation time function $\Delta t$ defined as the time required for the resin to cover a distance $\Delta l$ in each warp and weft bundle was determined as shown by Equation (13):

$$
\begin{aligned}
& \Delta t=-\frac{\mu \varphi_{\mathrm{cr}}}{k \cdot \frac{\partial P}{\partial n_{\text {axis }}}}\left(\Delta l+\frac{P_{\mathrm{c}}}{\frac{\partial P}{\partial n_{\text {axis }}}} \cdot \ln \left(1-\frac{\frac{\partial P}{\partial n_{\text {axis }}}}{P_{\mathrm{c}}} \cdot \Delta l\right)\right), \\
& \frac{\partial P}{\partial n_{\text {axis }}} \neq 0
\end{aligned}
$$

Because the $\partial P / \partial n_{\text {axis }}$ is 0 at an impregnation angle of $90^{\circ}$ for the impregnation of the warp bundles and at an impregnation angle of $0^{\circ}$ for the impregnation of the weft bundles, the intra-bundle impregnation time at the respective angles is denoted by Equation (14):

$\Delta t=-\frac{\mu \varphi_{\mathrm{cr}} \Delta l^{2}}{2 k P_{\mathrm{c}}}, \frac{\partial P}{\partial n_{\text {axis }}}=0$

The warp impregnation time $\Delta t_{\text {warp }}$ and the weft impregnation time $\Delta t_{\text {weft }}$ are obtained by substituting the material properties of each bundle, namely the macroscopic pressure gradient in each bundle direction (Equations (2) and (3)), and the impregnation distances for each bundle (Equations (11) and (12)) into Equations (13) and (14), respectively. The total intra-bundle resin impregnation time $\Delta t_{\text {total }}$ is specified by Equation (15):

$\Delta t_{\text {total }}=\Delta t_{\text {warp }}+\Delta t_{\text {weft }}$

The intra-bundle impregnation time increases with the impregnation angle for Pattern 1, whereas the reverse is true for Pattern 2. The minimum-void angle $\theta_{\min }$ is an angle, at which the relationship between the lengths of the intra-bundle resin impregnation times in Patterns 1 and 2 is reversed. In other words, an angle that satisfies the following equation is the minimum-void angle $\theta_{\min }$ (Equation (16)):

$\Delta t_{1}-\Delta t_{2}=0$

where $\Delta t_{1}$ and $\Delta t_{2}$ are the intra-bundle impregnation times for Patterns 1 and 2, respectively.

\subsection{Inter-bundle impregnation path and void formation}

Assuming that the resin flow velocity through the inter-bundle cavity is the same as the macroscopic flow velocity, the macroscopic resin flow velocity Un in the inter-bundle cavity in the impregnation direc tion can be expressed by Equation (17) based on the Darcy's law:

$U_{\mathrm{n}}=\frac{K_{\mathrm{n}}}{\mu \Phi_{\mathrm{n}}} \cdot \frac{\partial P}{\partial n}$

where $K_{\mathrm{n}}$ is the permeability in the resin impregnation direction, and $\Phi_{\mathrm{n}}$ is the sectional porosity of the fabric at the mold gap in the resin-impregnation direction. The subscript $n$ denotes the resin impregna- 
tion direction at the flow front. Considering the minuteness of the unit cell, if the resin flow velocity during the resin impregnation in the inter-bundle cavity is constant, the resin impregnation time in the inter-bundle cavity at an arbitrary angle $\Delta T_{\text {inter }}$ can be expressed as Equation (18):

$\Delta T_{\text {inter }}=\frac{L}{U_{\mathrm{n}}}=-\frac{\mu \Phi_{\mathrm{n}}\left(l_{\text {warp }} \cos \theta+l_{\text {weft }} \sin \theta\right)}{K_{\mathrm{n}} \frac{\partial P}{\partial n}}$

where $L$ is the length of the inter-bundle cavity in the flow direction, and $l_{\text {warp }}$ and $l_{\text {weft }}$ are the distances between the fiber bundles in the warp and weft directions, respectively (see Figure 6).

In addition, the resin impregnation of the inter-bundle cavity begins after the completion of that in the upstream transverse bundle shown in Figure $7 \mathrm{~b}$, and the impregnation time for the upstream transverse fiber bundle is added to the impregnation time for the inter-bundle path. As shown in Figure 7a, the resin impregnation of the transverse bundle begins at the two crimps (with an intra-bundle velocity $u_{\text {bundle }}$ ) and proceeds at the boundary between the upstream interbundle cavity and the transverse bundle (with a macroscopic velocity $\left.U_{\mathrm{n}}\right)$. Knowing the flow volume and the transverse bundle width, the impregnation time $\Delta T_{\text {bundle }}$ of the transverse bundle can be determined by Equations (19) and (20):

$$
\Delta T_{\text {bundle }}=\frac{w_{\text {bundle }} \Delta L_{\text {bundle }}}{u_{\text {bundle }} w_{\text {bundle }}+U_{n} \Delta L_{\text {bundle }}}
$$

where

$L_{\text {bundle }}= \begin{cases}\frac{l_{\text {weft }}}{2} & \text { for Pattern } 1 \\ \frac{l_{\text {warp }}}{2} & \text { for Pattern 2 }\end{cases}$

Here $w_{\text {bundle }}$ is the width of the transverse bundle, and ubundle can be calculated using Equation (1). From Equations (18) and (19), the total inter-bundle impregnation time $\Delta T_{\text {total }}$ can be obtained (Equation (21)):

$$
\Delta T_{\text {total }}=\Delta T_{\text {inter }}+\Delta T_{\text {bundle }}
$$

Assuming the regularity and continuity of the unit cell, the mean value of the void fraction $V_{\mathrm{v}}$ of the unit structure can be determined by Equation (22) [22]:

$V_{\mathrm{v}}=\left(1-\frac{\Delta t_{\text {total }}}{\Delta T_{\text {total }}}\right) \cdot \Phi$ where $\Phi$ is the macroscopic porosity of the fabric obtained from the ratio of the inter-bundle domain to the whole fabric area. The macroscopic porosity is obtained by measuring the ratio occupied by inter-bundle cavities by analyzing images of the fabric taken from above.

\section{Validation of the analytical prediction model 4.1. Void distribution}

The prediction model was applied to determine the void fraction. The material properties of the fabrics and resin used in this model are given in Tables 1 and 2. From fitting the experimental data, the geometric correction factor $C$ for the capillary pressure was calculated to be 2.4 for M155 and 1.4 for M200. In addition, a resin infusion point radius $r_{0}$ of $0.003 \mathrm{~m}$ and a gap between the molds $H$ of 0.0003 m were used for the model calculations. The macroscopic resin flow velocity and the pressure gradient values were calculated analytically (see Appendix A).

Using Equation (16), the minimum-void angle $\theta_{\min }$ in the prediction model was determined to be $53^{\circ}$ for M155 and $56^{\circ}$ for M200. Little deviations exist between the minimum-void angle values obtained by the prediction model and those determined by the experiment ( $49^{\circ}$ for M155 and 53 ${ }^{\circ}$ for M200) for both fabrics. The difference between the impregnation angles is very small for the elliptical shape indicating that the minimum-void angle $\theta_{\min }$ predicted by the model is in good agreement with the experimentally determined value. Figures 10 show the predicted void distributions for M155 and M200 demonstrating that void formation in the direction of the minimum-void angle is very difficult, and that the void distribution changes at the boundary of the minimum-void angle for both fabrics. This is in agreement with the experimental observations presented in Figure 5a and 5b.

\subsection{Relationship between the void fraction and the macroscopic resin flow velocity}

The analytically obtained curves of the void fraction as a function of the logarithm of the resin flow velocity for both fabrics are shown in Figures 11a and 11b, where the experimental results are also presented for comparison. The obtained theoretical and experimental results are in good agreement, thus validating the void fraction prediction model described in this work. 

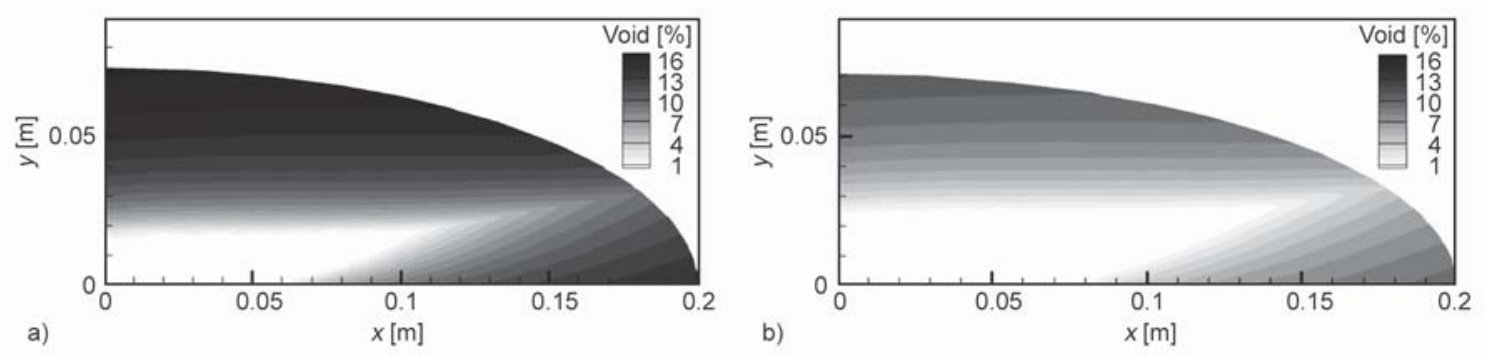

Figure 10. Void distributions produced by the single-point resin injection into the woven fabric determined by the void fraction prediction model: (a) M155, (b) M200

The curves of the void fraction as a function of the resin impregnation angle for both fabrics at a constant resin flow velocity are shown in Figures $11 \mathrm{c}$ and 11d. Capillary number $C a^{*}=0.00142$ and 0.000406 were used for M155 and M200, respectively. Due to a very low value of the resin flow velocity, the experimental results for M200 were not measurable at impregnation angles of 0 and $30^{\circ}$. At constant resin flow velocities, the void fraction was the largest at an impregnation angle of $0^{\circ}$ for both Patterns 1 and 2 and smallest at the minimum-void angle value. The deviation between the results predicted by the model and
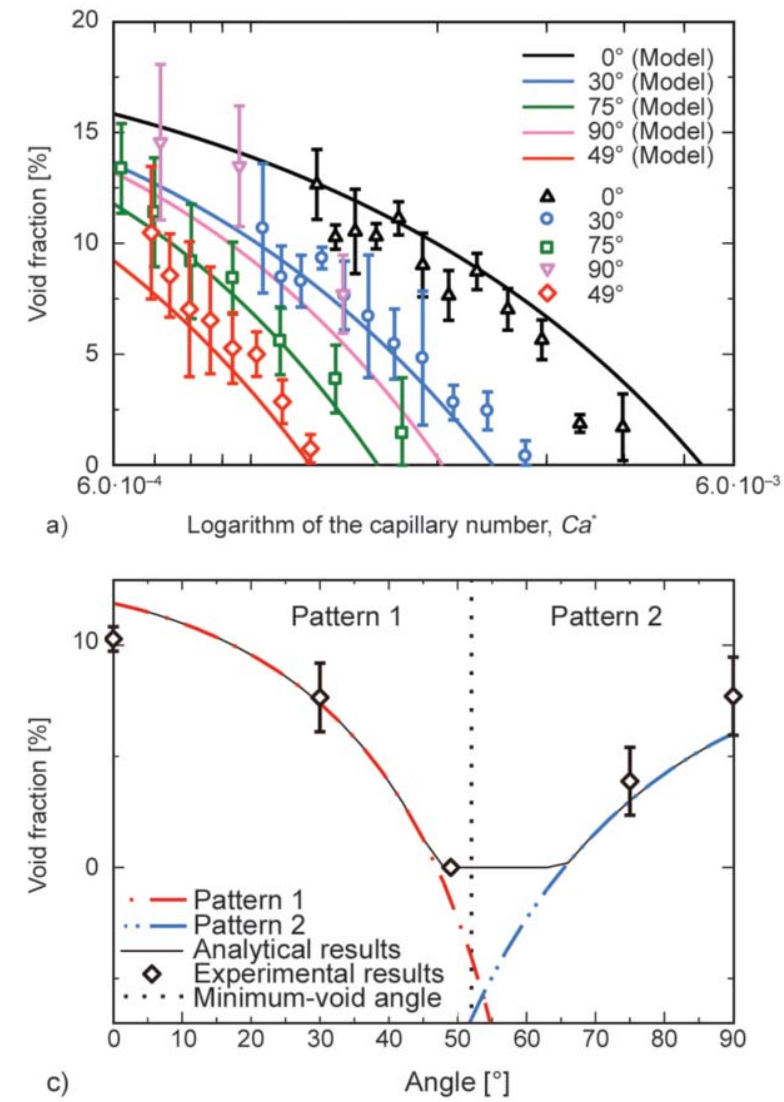

experimental results was due to experimental variation and the model being constructed with simplified fabric. However, as the void-formation trends could be reproduced, we think that it is useful for the constructed void-formation prediction model. The reason why the deviation between the experimental and theoretical values is large in places is thought to be that the impregnation differed from the ideal situation because of disordered impregnation caused by the creases and wrinkles of the fabric. Moreover, because the measured area was small, the difference between the void formation and non-formation increased. Further-
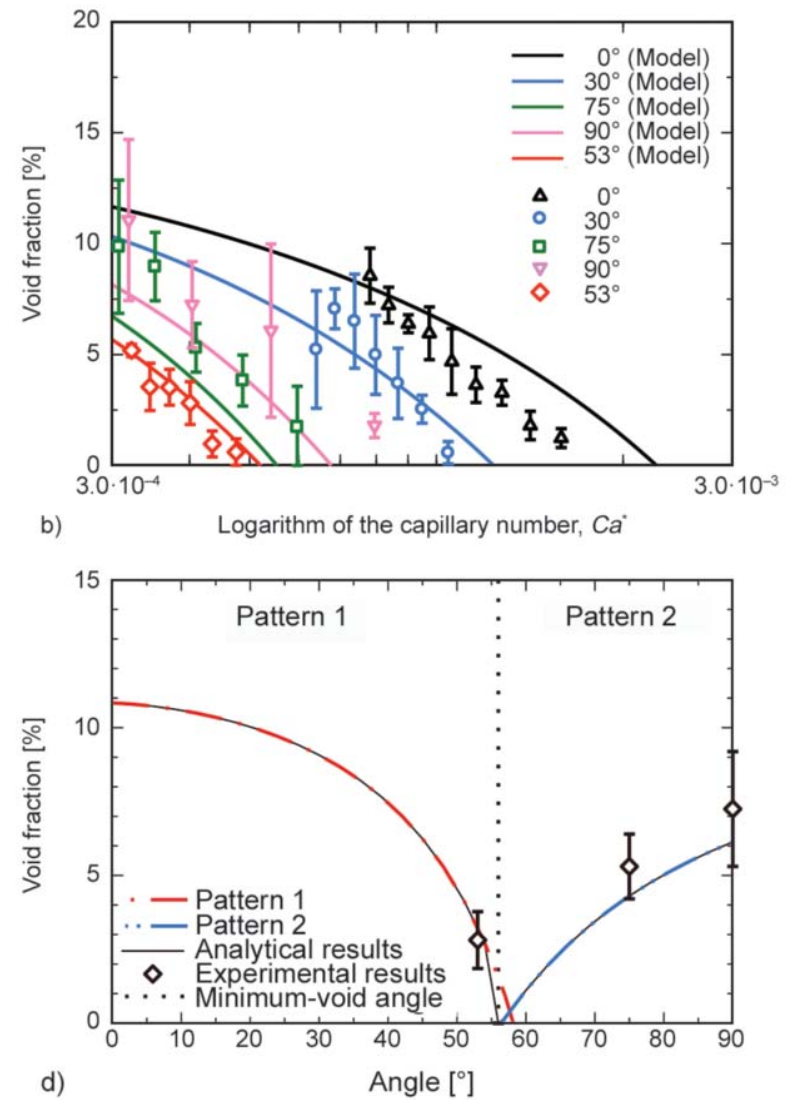

Figure 11. A comparison of the theoretical and experimental results. A relationship between the capillary number and the void fraction for different flow directions in (a) M155 and (b) M200. Void fraction as a function of the angle between the flow direction and the fiber orientation at a constant flow velocity in (c) M155 and (d) M200. 
more, deviations also occurred because the model for the present study was simplified and omitted the effect of the impregnation time for the crimps and perpendicular tilts and twists of the fiber bundles. Nonetheless, because as a trend the relationship between the impregnation angle and capillary number were virtually consistent between the experimental and predicted results, we consider the proposed model to be useful for predicting void formation.

\section{Conclusions}

Experimental 2D radial-injection VaRTM was performed using two types of an anisotropic plainwoven fabric. The void distributions during resin impregnation were obtained. We determined the relationship between the void fraction and the resin flow velocity for an impregnation direction inclined at an arbitrary angle to the fiber-bundling direction. Void formation was also demonstrated to be a function of the resin impregnation angle in the microscopic structure of the fabric. Our observations reveal that void formation varies with the resin impregnation angle and is minimized at the minimum-void angle, which corresponds to the border between the two void formation patterns. Based on the variations in the void formation pattern with the resin impregnation angle in the microscopic structure of the fabric, a theoretical model was developed for predicting the void fraction value at arbitrary values of the resin impregnation angle and velocity in the anisotropic fabric. This model can also be used to predict the minimum-void angle values, for which void formation is minimized. The model predictions were compared with the experimental results indicating a good agreement between them.

For simplicity, the present study was devoted to void formation in a single fabric layer. However, a realistic composite structure contains multiple layers, which may induce air trappings between the layers in addition to those existing within the layers. Therefore, further studies are required to develop an analytical model for predicting void formation in multiple layers. The proposed model would necessarily consider spatial uncertainties in mesoscale structures caused by overlapping layers.

\section{Appendix A. \\ Derivations of the macroscopic resin flow velocity and pressure gradient parameters for elliptical resin impregnation}

The experiments in this study were related to the 2D radial-injection VaRTM, in which the resin was infused from the center of the fabric and impregnated in the radial direction, as shown in Figure 1a. Therefore, the macroscopic resin flow velocity and the pressure gradient for elliptical resin impregnation parameters were derived for a comparison with the experimental results.

The 2D pressure governing equation for the resin impregnation by one-point injection can be obtained from Equation (A.1) [30, 31]:

$\frac{\partial^{2} P}{\partial x^{2}}+\frac{K_{\mathrm{y}}}{K_{\mathrm{x}}} \cdot \frac{\partial^{2} P}{\partial y^{2}}=0$

where $K_{\mathrm{x}}$ and $K_{\mathrm{y}}$ are the macroscopic permeabilities in the $x$ and $y$ directions, respectively (taking into consideration the sectional porosity).

By using the quasi-isotropic distances $\bar{x}$ and $\bar{y}$ determined by Equation (A.2), the domain in Equation (A.1) was modified into the quasi-isotropic one expressed by Equation (A.3) for a pressure field:

$\bar{x}=\left(\frac{K_{\mathrm{y}}}{K_{\mathrm{x}}}\right)^{\frac{1}{4}} x, \quad \bar{y}=\left(\frac{K_{\mathrm{x}}}{K_{\mathrm{y}}}\right)^{\frac{1}{4}} y$

$\frac{\partial^{2} P}{\partial \bar{x}^{2}}+\frac{\partial^{2} P}{\partial \bar{y}^{2}}=0$

By converting Equation (A.3) into polar coordinates, an ordinary differential equation containing a quasiisotropic radius $\bar{r}$ is obtained (Equation (A.4)):

$\frac{\mathrm{d}^{2} P}{\mathrm{~d} \bar{r}^{2}}+\frac{1}{r} \cdot \frac{\mathrm{d} P}{\mathrm{~d} \bar{r}}=0$

where the quasi-isotropic radius $\bar{r}$ is defined by (Equation (A.5)):

$\bar{r}=\sqrt{\bar{x}^{2}+\bar{y}^{2}}$

From Equation (A.4), the quasi-isotropic macroscopic pressure gradient is obtained for the boundary condition $P=0$ at a flow front $\bar{r}=\bar{r}_{\mathrm{f}}$ and $P=P_{\text {vac }}$ at an injection point $\bar{r}=\bar{r}_{0}$ as Equation (A.6):

$\frac{\mathrm{d} P}{\mathrm{~d} \bar{r}}=\frac{P_{\mathrm{vac}}}{\ln \bar{r}_{\mathrm{f}}-\ln \bar{r}_{0}} \cdot \frac{1}{\bar{r}}$ 
where $\bar{r}_{0}$ is the quasi-isotropic injection point determined by Equation (A.7) for an injection point radius $r_{0}$ and an angle $\varphi$ formed by the center point:

$\overline{r_{0}}=r_{0} \sqrt{\left(\frac{K_{\mathrm{y}}}{K_{\mathrm{x}}}\right)^{\frac{1}{2}} \cos ^{2} \varphi+\left(\frac{K_{\mathrm{x}}}{K_{\mathrm{y}}}\right)^{\frac{1}{2}} \sin ^{2} \varphi}$

The quasi-isotropic permeability $\bar{K}$ in the resin impregnation direction can be calculated by using Equation (A.8), whereas the quasi-isotropic resin flow velocity $\bar{U}$ is defined by Equation (A.9):

$\bar{K}=\sqrt{K_{\mathrm{x}} K_{\mathrm{y}}}$

$\bar{U}=-\frac{\bar{K}}{\mu} \cdot \frac{\mathrm{d} P}{\mathrm{~d} \bar{r}}$

Using the quasi-isotropic resin flow velocity calculated earlier, the real resin flow velocity value can be obtained. The coefficients used to convert the quasi-isotropic resin flow velocities in the $x$ and $y$ directions into the real flow velocities are $\left(K_{\mathrm{x}} / K_{\mathrm{y}}\right)^{1 / 4}$ and $\left(K_{\mathrm{y}} / K_{\mathrm{x}}\right)^{1 / 4}$, respectively, whereas the tensor $\mathbf{c}_{\mathrm{K}}$ used to convert the quasi-isotropic resin flow velocity into the real flow velocity at an arbitrary impregnation angle is defined by Equation (A.10):

$\mathbf{c}_{\mathrm{K}}=\left[\begin{array}{cc}\left(\frac{K_{\mathrm{x}}}{K_{\mathrm{y}}}\right)^{\frac{1}{4}} & 0 \\ 0 & \left(\frac{K_{\mathrm{y}}}{K_{\mathrm{x}}}\right)^{\frac{1}{4}}\end{array}\right]$

The real resin flow velocity for an arbitrary angle in terms of the tensor $\mathbf{c}_{\mathrm{K}}$ can be determined using Equation (A.11):

$\mathrm{U}=\mathbf{c}^{\mathrm{T}} \mathbf{c}_{\mathrm{K}} \mathbf{c} \overline{\mathrm{U}}$

where $\mathbf{c}$ is the transformation matrix. From Equation (A.11), the real resin flow velocity $U_{\mathrm{n}}$ in the resin impregnation direction can be expressed as Equation (A.12):

$U_{\mathrm{n}}=\left(\left(\frac{K_{\mathrm{x}}}{K_{\mathrm{y}}}\right)^{\frac{1}{4}} \cos ^{2} \theta+\left(\frac{K_{\mathrm{y}}}{K_{\mathrm{x}}}\right)^{\frac{1}{4}} \sin ^{2} \theta\right) \bar{U}$

Here the permeability $K_{\text {aniso }}$ and flow velocity $U_{\text {aniso }}$ values at an arbitrary angle in anisotropic fabrics are expressed using the macroscopic pressure gradient of the resin impregnation direction $\partial P / \partial n$ [32] (Equations (A.13) and (A.14)):

$$
K_{\text {aniso }}=K_{\mathrm{x}} \cos ^{2} \theta+K_{y} \sin ^{2} \theta
$$

$$
U_{\text {aniso }}=-\frac{K_{x} \cos ^{2} \theta+K_{y} \sin ^{2} \theta}{\mu} \cdot \frac{\partial P}{\partial n}
$$

Assuming that the real resin flow velocity $U_{\mathrm{n}}$ is the same as the flow velocity $U_{\text {aniso }}$ in the flow direction, $\partial P / \partial n$ can be expressed as Equations (A.15):

$\frac{\partial P}{\partial n}=\bar{K} \frac{\left(\left(\frac{K_{\mathrm{x}}}{K_{\mathrm{y}}}\right)^{\frac{1}{4}} \cos ^{2} \theta+\left(\frac{K_{\mathrm{y}}}{K_{\mathrm{x}}}\right)^{\frac{1}{4}} \sin ^{2} \theta\right)}{K_{\mathrm{x}} \cos ^{2} \theta+K_{\mathrm{y}} \sin ^{2} \theta}$

\section{References}

[1] Mallick P. K.: Fiber-reinforced composites. CRC Press, Boca Raton (1993).

[2] Gibson R. F.: Principles of composite material mechanics. CRC Press, Boca Raton (2011).

[3] Advani S. G., Sozer E. M.: Process modeling in composites manufacturing. CRC Press, Boca Raton (2002).

[4] Matsuzaki R., Kobayashi S., Todoroki A., Mizutani Y.: Flow control by progressive forecasting using numerical simulation during vacuum-assisted resin transfer molding. Composites Part A: Applied Science and Manufacturing, 45, 79-87 (2013).

DOI: 10.1016/j.compositesa.2012.09.014

[5] Matsuzaki R., Kobayashi S., Todoroki A., Mizutani Y.: Control of resin flow/temperature using multifunctional interdigital electrode array film during a VaRTM process. Composites Part A: Applied Science and Manufacturing, 42, 782-793 (2011).

DOI: $10.1016 /$ j.compositesa.2011.03.004

[6] Huang H., Talreja R.: Effects of void geometry on elastic properties of unidirectional fiber reinforced composites. Composites Science and Technology, 65, 1964 1981 (2005).

DOI: $10.1016 /$ j.compscitech.2005.02.019

[7] Varna J., Joffe R., Berglund L. A., Lundström T. S.: Effect of voids on failure mechanisms in RTM laminates. Composites Science and Technology, 53, 241-249 (1995). DOI: $10.1016 / 0266-3538(95) 00024-0$

[8] Ghiorse S. R.: Effect of void content on the mechanical properties of carbon/epoxy laminates. SAMPE Quarterly, 24, 54-59 (1993).

[9] Lee D. H., Lee W. I., Kang M. K.: Analysis and minimization of void formation during resin transfer molding process. Composites Science and Technology, 66, 3281-3289 (2006).

DOI: $10.1016 /$ j.compscitech.2005.07.008

[10] Johnson R. J., Pitchumani R.: Flow control using localized induction heating in a VaRTM process. Composites Science and Technology, 67, 669-684 (2007). DOI: $10.1016 /$ j.compscitech.2006.04.012 
[11] Matsuzaki R., Kobayashi S., Todoroki A., Mizutani Y.: Full-field monitoring of resin flow using an area-sensor array in a VaRTM process. Composites Part A: Applied Science and Manufacturing, 42, 550-559 (2011). DOI: $10.1016 /$ j.compositesa.2011.01.014

[12] Patel N., Rohatgi V., Lee L. J.: Micro scale flow behavior and void formation mechanism during impregnation through a unidirectional stitched fiberglass mat. Polymer Engineering and Science, 35, 837-851 (1995). DOI: $10.1002 /$ pen.760351006

[13] Schell J. S. U., Deleglise M., Binetruy C., Krawczak P., Ermanni P.: Numerical prediction and experimental characterisation of meso-scale-voids in liquid composite moulding. Composites Part A: Applied Science and Manufacturing, 38, 2460-2470 (2007).

DOI: $10.1016 /$ j.compositesa.2007.08.005

[14] Lundström T. S., Gebart B. R.: Influence from process parameters on void formation in resin transfer molding. Polymer Composites, 15, 25-33 (1994).

DOI: $10.1002 /$ pc.750150105

[15] Chen Y-T., Davis H. T., Macosko C. W.: Wetting of fiber mats for composites manufacturing: I. Visualization experiments. AIChE Journal, 41, 2261-2273 (1995). DOI: $10.1002 /$ aic.690411009

[16] Yamaleev N., Mohan R.: Effect of the phase transition on intra-tow flow behavior and void formation in liquid composite molding. International Journal of Multiphase Flow, 32, 1219-1233 (2006).

DOI: $10.1016 /$ j.ijmultiphaseflow.2006.05.011

[17] Liu B., Bickerton S., Advani S. G.: Modelling and simulation of resin transfer moulding (RTM)-gate control, venting and dry spot prediction. Composites Part A: Applied Science and Manufacturing, 27, 135-141 (1996). DOI: 10.1016/1359-835X(95)00012-Q

[18] Restrepo O., Hsiao K-T., Rodriguez A., Minaie B.: Development of adaptive injection flow rate and pressure control algorithms for resin transfer molding. Composites Part A: Applied Science and Manufacturing, 38, 1547-1568 (2007).

DOI: 10.1016/j.compositesa.2007.01.005

[19] Phelan F. R.: Simulation of the injection process in resin transfer molding. Polymer Composites, 18, 460-476 (1997). DOI: $\underline{10.1002 / p c .10298}$

[20] Gokce A., Hsiao K-T., Advani S. G.: Branch and bound search to optimize injection gate locations in liquid composite molding processes. Composites Part A: Applied Science and Manufacturing, 33, 1263-1272 (2002). DOI: 10.1016/S1359-835X(02)00047-7

[21] Park C. H., Lebel A., Saouab A., Bréard J., Lee W. I.: Modeling and simulation of voids and saturation in liquid composite molding processes. Composites Part A: Applied Science and Manufacturing, 42, 658-668 (2011). DOI: $\underline{10.1016 / \text { j.compositesa.2011.02.005 }}$
[22] Matsuzaki R., Seto D., Todoroki A., Mizutani Y.: Void formation in geometry-anisotropic woven fabrics in resin transfer molding. Advanced Composite Materials, 23, 99-114 (2013). DOI: $10.1080 / 09243046.2013 .832829$

[23] Matuzaki R., Seto D., Naito M., Todoroki A., Mizutani Y.: Analytical prediction of void formation in geometrically anisotropic woven fabrics during resin transfer molding. Composites Science and Technology, 107, 154-161 (2015).

DOI: $10.1016 /$ j.compscitech.2014.12.013

[24] Matsuzaki R., Seto D., Todoroki A., Mizutani Y.: In situ void content measurements during resin transfer molding. Advanced Composite Materials, 22, 239-254 (2013). DOI: $10.1080 / 09243046.2013 .801822$

[25] Kang M. K., Lee W. I., Hahn H. T.: Formation of microvoids during resin-transfer molding process. Composites Science and Technology, 60, 2427-2434 (2000). DOI: $10.1016 / \mathrm{S} 0266-3538(00) 00036-1$

[26] Rohatgi V., Patel N., Lee L. J.: Experimental investigation of flow-induced microvoids during impregnation of unidirectional stitched fiberglass mat. Polymer Composites, 17, 161-170 (1996). DOI: $10.1002 / \mathrm{pc} .10601$

[27] Erickson D., Li D., Park C. B.: Numerical simulations of capillary-driven flows in nonuniform cross-sectional capillaries. Journal of Colloid and Interface Science, 250, 422-430 (2002).

DOI: $10.1006 /$ jcis.2002.8361

[28] Gebart B. R.: Permeability of unidirectional reinforcements for RTM. Journal of Composite Materials, 26, 1100-1133 (1992). DOI: $10.1177 / 002199839202600802$

[29] Ahn K. J., Seferis J. C., Berg J. C.: Simultaneous measurements of permeability and capillary pressure of thermosetting matrices in woven fabric reinforcements. Polymer Composites, 12, 146-152 (1991).

DOI: $10.1002 /$ pc. 750120303

[30] Adams K. L., Russel W. B., Rebenfeld L.: Radial penetration of a viscous liquid into a planar anisotropic porous medium. International Journal of Multiphase Flow, 14, 203-215 (1988).

DOI: $10.1016 / 0301-9322(88) 90006-7$

[31] Vanegas J. D., Patiño I. D., Vargas C. A.: Boundary element approaches for filling simulations of anisotropic reinforced preforms used in the resin transfer molding process. Journal of Composite Materials, 48, 26032629 (2014).

DOI: $10.1177 / 0021998313501917$

[32] Wang T. J., Wu C. H., Lee L. J.: In-plane permeability measurement and analysis in liquid composite molding. Polymer Composites, 15, 278-288 (1994).

DOI: $10.1002 /$ pc. 750150406 